\title{
Hot Jupiters and the evolution of stellar angular momentum
}

\author{
Cilia Damiani ${ }^{1}$ and Antonino F. Lanza ${ }^{1}$ \\ ${ }^{1}$ INAF- Osservatorio Astrofisico di Catania, \\ Via Santa Sofia, 78, 95123, Catania, Italy \\ email: damiani@oact.inaf.it
}

\begin{abstract}
A close-in massive planet affects the angular momentum of its host star through tidal and magnetic interactions. The transiting planets allow us to study the distribution of the spin and orbital angular momenta in star-planet systems. Considering a sample of about 70 systems, we find that stars having an effective temperature between 6000 and $6700 \mathrm{~K}$ and a rotation period shorter than 10 days show a rotation synchronized with the orbit of their hot Jupiters or have a rotation period twice the orbital period of their planets. Such rotational behaviours cannot be explained on the basis of tidal interactions alone. Besides, the gyrochronology relationship for those systems holds if an angular momentum loss rate smaller by about 30 percent than in stars without hot Jupiters is assumed.
\end{abstract}

Keywords. planetary systems, planet-star interactions, stars: rotation

\section{Introduction}

Remarkable interactions between a close-in massive planet and its host stars are expected, both as a consequence of tides and reconnection between planetary and stellar magnetic fields. In the present study, updating the work of Lanza (2010), we give observational evidence of such interactions and show that they affect the evolution of stellar angular momentum. We limit ourselves to the sample of transiting systems so that the inclination of the rotation axis can be estimated, assuming that the stellar spin and orbital angular momentum are aligned. As a consequence, we exclude from the study systems for which a notable eccentricity or obliquity have been measured. As of September 2010, this yields 73 systems out of the 97 confirmed transiting planetary systems $\dagger$.

\section{Properties of transiting planetary systems}

As a measure of the synchronization of a planet-harbouring star, we adopt the ratio $n / \Omega=P_{\text {rot }} / P_{\text {orb }}$ between the rotational period of the star and the orbital period of the planet. Here we focus on the relation between $n / \Omega$ and the effective temperature of the star, plotted in Fig.1.a. Although there is a large scatter for $T_{\text {eff }}<6000 \mathrm{~K}$, there is a general trend towards synchronization with increasing effective temperature. For $T_{\text {eff }} \geqslant 6000 \mathrm{~K}$, two subgroups of systems can be identified, those that are close to $n / \Omega=1$ or to $n / \Omega=2$ and those showing $n / \Omega$ remarkably greater than 2 . These two groups appear to be differentiated by their rotation period, with all systems with $P_{\text {rot }} \leqslant 10$ days having $n / \Omega<2$, with the exception of WASP-18 and WASP-24. A Kolmogorov-Smirnov test confirms the statistical difference of the two populations, and their non-uniform distribution in $n / \Omega$, thus confirming the result of Lanza (2010).

$\dagger$ See e.g. http://www.inscience.ch/transits/ 

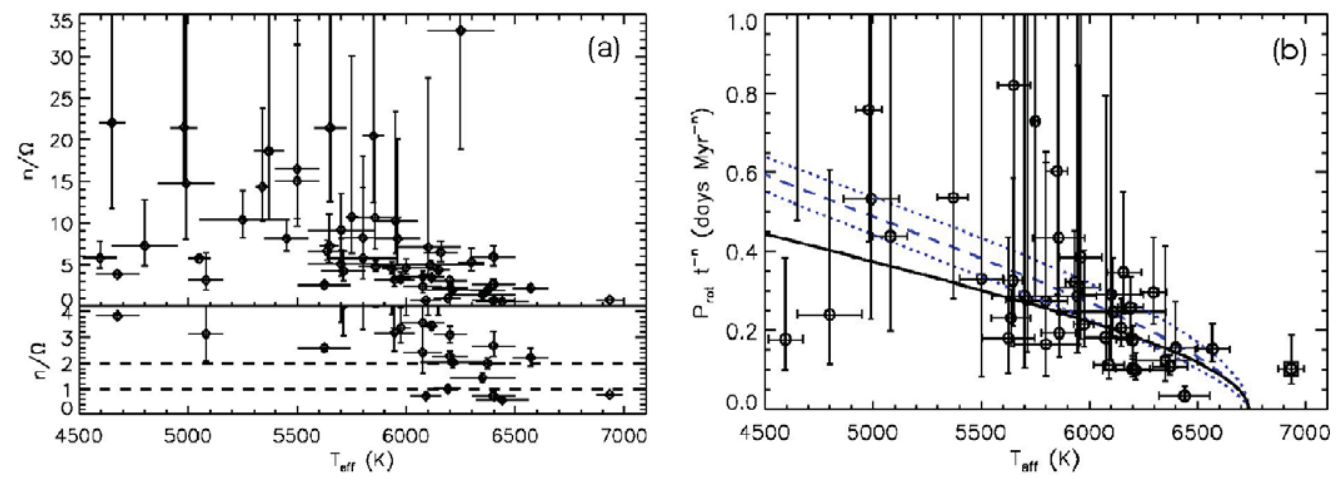

Figure 1. (a) Upper panel: the synchronization parameter $n / \Omega$ vs. the effective temperature of the star $T_{\text {eff }}$ in transiting planetary systems. Lower panel: an enlargement of the lower portion of the upper panel, to better show the correlation close to $n / \Omega=1$ and $n / \Omega=2$. (b) Age-normalized rotation period $P_{\text {rot }} t^{-n}$, with $\mathrm{n}=0.5189$, vs $T_{\text {eff }}$, for the systems with a known age estimate of the host star. The relationship found by Barnes (2007) for stars without hot Jupiters is plotted as a dashed blue line, together with its $3 \sigma$ errors as dotted lines. The solid thick line plots the best one-parameter fit. OGLE-TR-L9 (square symbol) was not included in the study.

According to Barnes (2007), for stars without hot Jupiters, the rotation period $P_{\text {rot }}$ in days and the age $t$ in $\mathrm{Myr}$ are related to the $B-V$ colour index by the formula $P_{\text {rot }} t^{-n}=a[(B-V)-0.4]^{b}$ with prescribed values for $n, a$, and $b$. We plot in Fig.1.b $P_{\text {rot }} t^{-n}$ vs $T_{\text {eff }}$, converting the $B-V$ index into effective temperature with the calibration by Bessel (1979). The relation by Barnes (2007) is plotted as a dashed blue line, together with its $3 \sigma$ error range as dotted lines. The goodness of fit is almost null for stars with hot Jupiters. On the other hand, the best fit for $a$ (linked to the angular momentum loss rate) is smaller by a factor 0.7 for our sample of transiting planetary systems (solid line).

This leads to the conclusion that on average, the stars with transiting close-in planets are faster rotators than stars without hot Jupiters, for a given age and temperature. Thus, the angular momentum loss rate of the former is lower than that of the latter (cf. Cohen et al. 2010).

\section{Conclusions}

We have analysed the rotation of stars harbouring transiting hot Jupiters and found a general trend towards synchronization with increasing effective temperature. We have shown that the rotation periods of the planet-hosting stars are, on the average, a factor of 0.7 shorter than those of the stars without planets of the same age. It is thus likely that both tides and magnetic effects are simultaneously at work to shape the distribution of angular momentum and its evolution in stars harbouring hot Jupiters.

Finally, we note that gyrochronology may not be suitable for estimating the age of late-type stars with close-in giant planets, especially if they have $T_{\text {eff }} \geqslant 6000 \mathrm{~K}$ and/or are rotating with a period shorter than $\approx 10$ days, because their rotational evolution can be remarkably different from that of stars without hot Jupiters.

\section{References}

Barnes, S. A. 2007, ApJ, 669, 1167

Bessell, M. S. 1979, PASP, 91, 589

Cohen, O., et al. 2010, ApJ, 723, L64

Lanza, A. F. 2010, A\& $A$, 512, A77 